\title{
Stems of Hypothetical Plants at the Landing Site of Venera-14
}

\author{
Leonid V. Ksanfomality \\ 84/32 Profsoyuznaya str., Space Research Institute, Moscow, 117997, Russia \\ *Corresponding Author: leksanf@gmail.com
}

Copyright (C) 2014 Horizon Research Publishing. All rights reserved.

\begin{abstract}
Presented in paper are the results of a study of hypothetical flora of Venus around the VENERA-14 lander. Because of the previous experience of searching for objects of hypothetical flora and fauna of Venus, successful processing of more complex panoramas of the VENERA-14 lander has been performed revealing multiple "stems" that should be added to the hypothetical flora of the planet. They are thin knotty vertical trunks that have a thickness of $0.3-3$ $\mathrm{cm}$ and are 0.2 to $0.5 \mathrm{~m}$ or more in height. On color panoramas, they look black. On close objects, one can see that the "stem" at the top end is provided with a large bulge, a "burgeon" or "flower" that is $2-8 \mathrm{~cm}$ in diameter; and other have petals surrounding a bright center. At the base of the stems, there are features that resemble leaves in a quatrefoil. Although the "stems" are numerous in the panoramas on both sides of VENERA-14 (where there are approximately eight stalks rising from cracks on the rocky ground), in the VENERA-13 panoramas, the "stems" are rare. Perhaps an object of the "stem" type is apparent at the landing site of VENERA-9. Probably, the "stems" are widespread on the planet, because the landing distances between the three different VENERA missions were 900 and $4500 \mathrm{~km}$. In general, hypothetical signs of flora on Venus go far beyond the direct results of the VENERA missions.
\end{abstract}

Keywords Extraterrestrial Life, Hypothetical Venus Flora, Terramorphism, VENERA Missions

\section{Introduction. Some Data on the VENERA Experiments}

The quest for hypothetical flora on Venus is based on analysis of the images of the landing site of the VENERA-13 and VENERA-14 landers. What was found are numerous vertically standing knotty black stems with heights of up to half a meter. Due to the availability of up to eight duplicates of the images obtained and their low level of masking noise, the VENERA panoramas permit identifying and exploring some new types of hypothetical life forms of Venus. Specifically, stems are the most numerous group of samples of hypothetical flora. The first stem object was detected due to its being close to the entrance of the TV camera, and the remaining was detected by similarities in their shapes and positions to the first stem.

The first group of publications that related to hypothetical signs of life on the planet Venus [1-3] refers to data that was obtained by the VENERA-9 and VENERA-13 landers. The results of a series of Soviet VENERA missions are the only existing observations of Venus' surface, and they were conducted in 1975 and 1982 [1,3]. The experiments were of extreme technical complexity.

Over the years, they have not been repeated by any Russian, NASA or other space agencies.

The thematic issue of "Kosmicheskiye Issledovaniya", V. XXI, No. 2-3, 1983, presented the main results of the VENERA-13 and -14 missions. The methodology of the television experiments on the surface of Venus and the date and list of the experimental data have been discussed in detail in $[1,3,4]$ and are not repeated here. Some remarks on the critical features of the treatment used are given below.

The lander VENERA-13 (March 1, 1982) worked longer than the others $(127 \mathrm{~min}$.) The coordinates of the lander landing site were $7.5^{\circ} \mathrm{S}, 303.5^{\circ} \mathrm{E}$, and its height above the level of radius $6051 \mathrm{~km}$ was $1.9 \mathrm{~km}[1,2]$. The temperature was $735 \mathrm{~K}\left(462^{\circ} \mathrm{C}\right)$ and the pressure was $8.87 \mathrm{MPa}$, which corresponds to the atmospheric density $59.5 \mathrm{~kg} / \mathrm{m}^{3}$, with the composition $\mathrm{CO}_{2}(96.5 \%)$ and $\mathrm{N}_{2}(3.5 \%)$. The local time was 10:00 am, and the solar zenith was at an angle of $37^{\circ}$. Illumination by the diffused sunlight was 3-3.5 kLux.

The lander VENERA-14 (March 5, 1982) sank at the equatorial zone at $13^{\circ} \mathrm{S}, 310^{\circ} \mathrm{E}$, and the landing site's height was $1.3 \mathrm{~km}$ above the radius of $6051 \mathrm{~km}$. The measured physical conditions were as follows: temperature $738 \mathrm{~K}$, pressure of $9.47 \mathrm{MPa}$ and atmospheric density approximately $65 \mathrm{~kg} / \mathrm{m}^{3}$. Gas analyzers repeated that the atmosphere is composed almost entirely of $\mathrm{CO}_{2}(96.5 \%)$ and $\mathrm{N}_{2}(3.5 \%)$. Local time was also at approximately $10 \mathrm{am}$, with a solar zenith angle of $36^{\circ}$. (For more details see $[3,4]$ ) The scene illumination reached $3.5 \mathrm{kLux}[1,5]$. In both cases, the transmission of images began with a one minute delay after landing, to prevent dust on the optical surfaces.

Detailed information on the planet surface and geology is 
given in $[6,7]$

Views of the planet's surface at the landing sites of both landers are shown in Fig.1. In the first case, the view was crushed soil with stone slabs, and in the second case, it was plates of lithified deposits or traces of ancient volcanic eruptions.
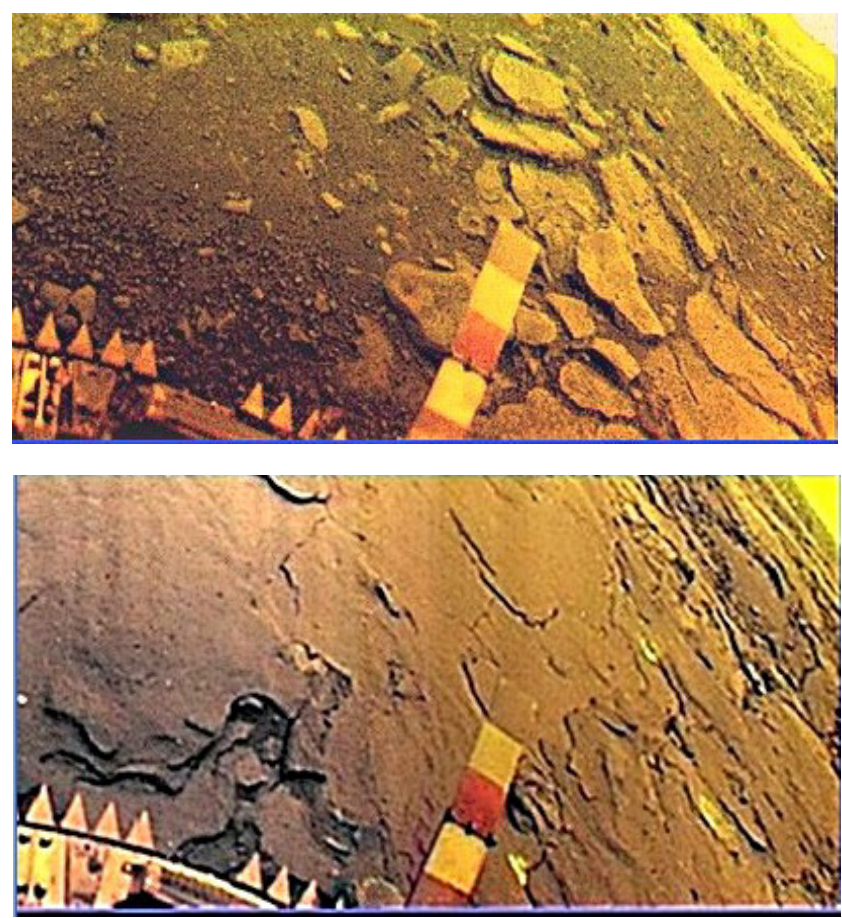

Figure 1. Planet's surface at the landing sites of VENERA-13 (left side) and VENERA-14. Geometric distortions are not corrected.

Physical properties of the surface at the VENERA -14 landing site (March 1982) were presented in [8].

At the time of this writing, two years have elapsed since the submission of the first manuscript for publication that was devoted to hypothetical signs of life on the planet Venus $[1,2]$. Discovered new objects have gradually been accumulated. These objects hypothetically have characteristics of living creatures - flora or fauna. At the initial stage of the investigation, mainly panoramas of VENERA -9 and - VENERA -13 were used, in which relatively large objects were found, such as "scorpion", "mushroom", "bear-cub" and "owl" [1-3, 8]. When experience using image processing was accumulated, the VENERA-14 panorama allowed an approach to the finer details. An important role was played by additional image processing, image geometric correction and the presence of up to eight duplicates of images that were obtained with good quality and low levels of noise. This arrangement enabled the selection and staking of their fragments. As a result, it managed to find and learn about a few new types of hypothetical living creatures at the VENERA-14 landing site.

Of importance is a question about the sources of energy for the hypothetical Venusian inhabitants. The interest in the proposed autotrophic flora of the planet as a source of the existence of its fauna was noted in [2, 3, 9]. It is natural to assume that, like on the Earth, the Venusian fauna is heterotrophic, and the source of its existence is hypothetical autotrophic flora. Although the direct rays of the Sun, as a rule, do not reach the surface of the planet, there is enough light for photosynthesis of the Earth-like type there. In the case of the Earth, a diffuse illumination of 0.5-5 kLux is sufficient for photosynthesis even in the depths of the dense forests. The measured illuminance on Venus is of the same order, at the range of 0.4 to 9 kLux. Of course, photosynthesis at high temperatures and in a non-oxidizing environment should be based on a completely different, unknown biophysical mechanism. Some information on the hypothetical flora of the planet is presented below.

\section{Stem at the Landing Buffer of the VENERA-14}

In input images, a "stem" resembles only a thin scratch, but it is repeated at all panoramas and in the same place (Fig. 2 ). When processed the "stems" are vertically arranged thin knotty trunks, which are $0.3-2 \mathrm{~cm}$ thick and $0.2-0.5 \mathrm{~m}$ (and more) tall. On color panoramas, they look black. The first object that was detected, which is a "stem" (Fig.3), has a large bulge at the top end, a "burgeon", with a lighter center. The first detected "stem" was mentioned already in the previous paper [10] in our magazine. Below are other sites where the "burgeon" is observed in development. The "stem" is located close to the camera. At the "stem's" base, on the surface, there is a visible group of details that resembles a quatrefoil. Each of the "leaves" has a size of approximately 5-10 cm, and possibly, they have a radial structure (Fig. 3). In the vicinity of VENERA-14, the number of stems at the panorama is approximately eight. All of the "stems" are placed vertically, with the exception of one of the largest, which bends to the surface.

To find the height $z$ of the stem in Figure 3, one should use geometric relations and a photoplan (because, on the original panoramas, the distances are significantly distorted). An exact photoplan of the landing site of VENERA-14 is currently being finalized and is not shown here. The input window of the TV camera is located at a height of $h=90 \mathrm{~cm}$, the distance $a$ from the projection point of the TV-camera lens onto the surface, to the base of the stem is approximately $40 \mathrm{~cm}$, and the top of the stem is projected onto the surface details, roughly at the distance of $b=75 \mathrm{~cm}$. If the stem is placed vertically, from the right triangle, then the angle $\alpha$ at its apex is found to be $\operatorname{tg} \alpha=b / h$, and the stem height is $z=$ $(b-a) / \operatorname{tg} \alpha=42 \mathrm{~cm}$. An error is possible as the ground surface is uneven.

All of the detected stems are thin and apparently knotted. However, perhaps there is one exception. In the peripheral part of the panorama of VENERA-9, there is an object that could be a thick stem (Figure 4). The image shows its light spotted top. 


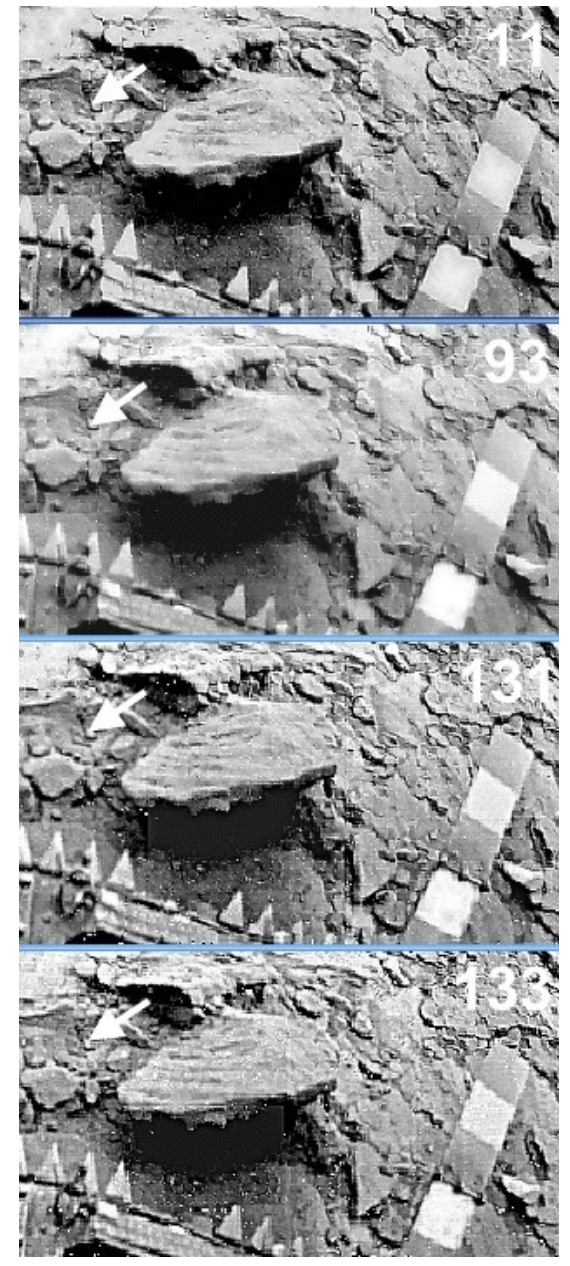

Figure 2. Unprocessed fragments of the image surface of the planet at the landing site of VENERA-14. A "stem" is shown by the arrow.

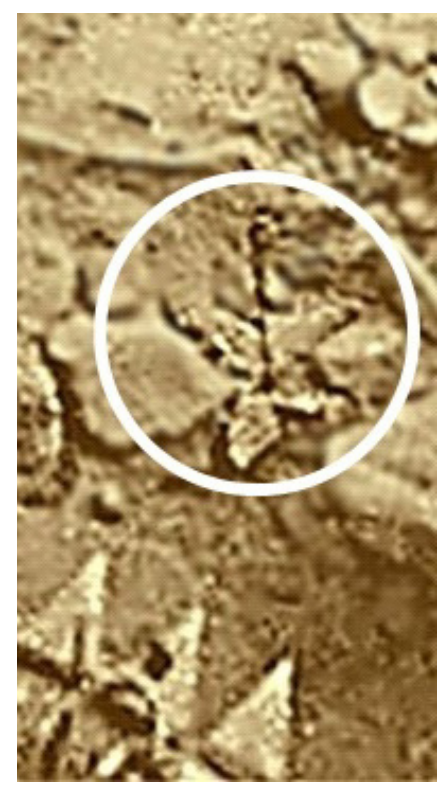

Figure 3. The first found object of the "stem" type is a thin vertically arranged knotty trunk that has a height of approximately $42 \mathrm{~cm}$ and a thickening ("burgeon") on the top. The "stem" is located at a distance of approximately $40 \mathrm{~cm}$ from the landing buffer of the VENERA-14 lander and is seen from above.

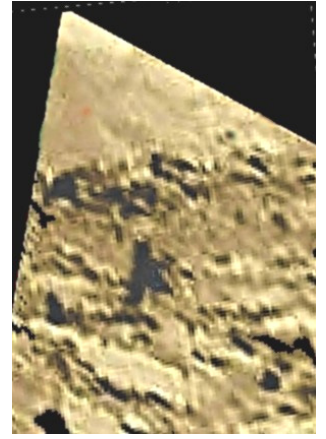

Figure 4. A vertical dark object at the center of the fragment of the VENERA-9 panorama could be a thickened "stem" with a light top.

Its height is approximately half a meter, the thickness of the stem is approximately $5-8 \mathrm{~cm}$, and the nodes are not visible. However, we should be reminded that the low resolution of the VENERA-9 images does not permit making firm conclusions. Other stems on the panorama of the VENERA-9 panorama were not found, perhaps for the same reason.

\section{Stems with Flowers}

To search for other stems, an additional processing of the VENERA-13 and -14 panoramas has been made to improve the clarity of the details. In some of the cases, the correction has been made of geometrically distorted panoramas, similar to in Fig. 1.

Because of the image correction, the "stems" are becoming almost vertical, and they stand out from the cracks in the rocks. In addition, the stems stand out due to their mutual parallelism. Black and white panoramas of VENERA-14 series; groups 1, 6 and 9, 13 (of camera 1) and 3,5 , and 7, 11 (of camera 2); and the "red" panoramas of the same series (besides defect series 7) were used. More information about the properties of the panoramas is presented in $[1,3,4]$. "Green" panoramas are difficult to use because they are noisier. The synthetic color panorama was used to obtain some information about the colors of the objects.

The clarity of details is different in different panoramas. Large but distant stems, perhaps more than half a meter in height, appear to be found in the left-hand edge of the panorama V14-6, at a distance of more than $3 \mathrm{~m}$ from the TV-lens; thus, the details of their structure are not seen. Several distant "stems" were found in the right part of the panorama V14-13. In all of the cases, the bases of the "stems" were located in crevices between stones. All of the stems that were found are solitary.

Unlike VENERA-14, at the VENERA-13 landing site, only one or two such objects were found, for which the base of the "stems", similar to in Figure 3, were in a crack between the stones. This circumstance can be important because the soil here is mainly fragmented, but the stems there were not found. This interesting object is shown in 
Figure 5, which presents four consecutive images of a knotty stem that was found in the VENERA-13 panoramas.
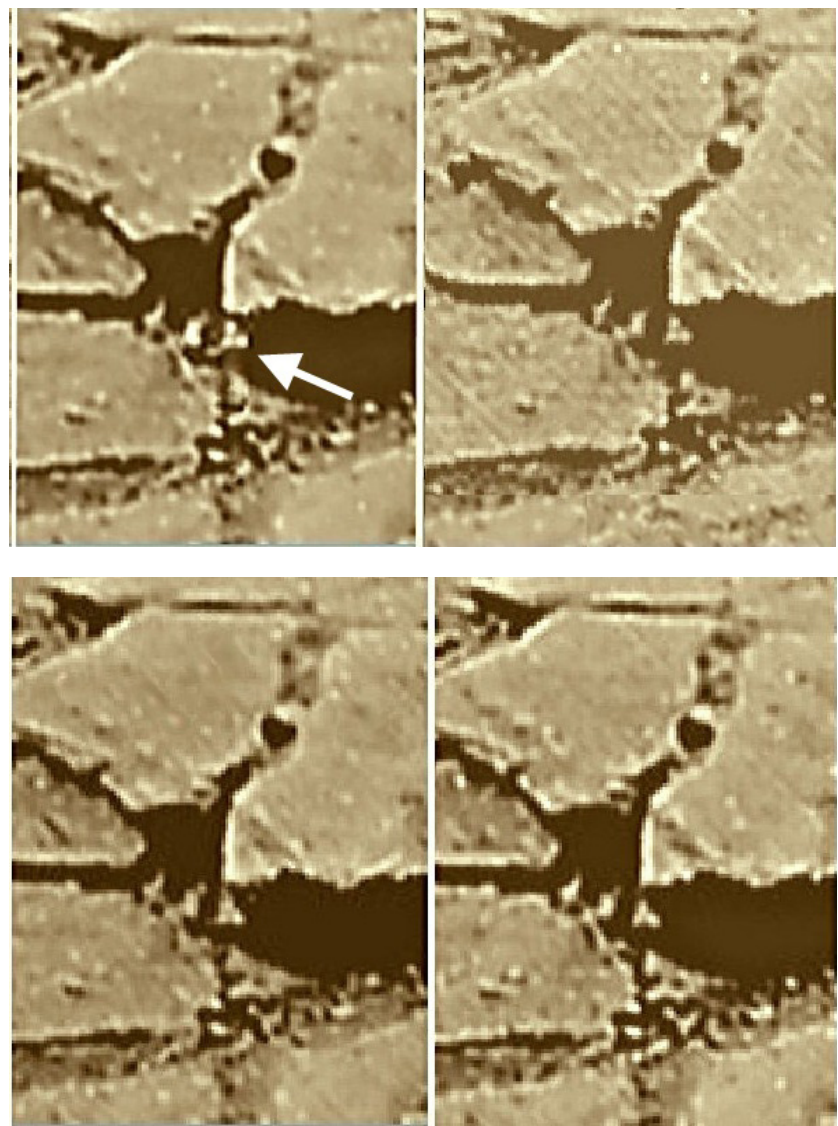

Figure 5. In the foreground - the "stem" at the landing site of VENERA-13 ( 4 consecutive images obtained within 1.5 hours).

However, the "stem" in Fig. 5 is lower than in Fig. 3; it is more distant, and the stem itself is not easy to notice, although there are eight distinct images (duplicates), which allows for batch processing. The attention is drawn to the top of the stem, which appears in Figure 5 as a triad of bright dots that are visible on all of the original high-contrast images. The position of the triad is not identical in successive frames. It varies slightly with respect to the adjacent light-colored stone on top of it (Figure 5). This change could arise from the swinging of the triad by the wind.

A clearer picture of the stem is highlighted by the circle in Figure 6. The top of the "stem" is more complex than the triad (or bud in Figure 3). The object is visible from above, and its height, which is found by its position on the photoplan, is only approximately $20-30 \mathrm{~cm}$ at the base in the crack between the stones. At its base, there is a group of four bright details, similar to the "quatrefoil" leaves shown in Figure 3; that appears to be associated with the stem, also.

It has been suggested that the complex structure of the top of the stem is an opened burgeon. When processing the image with a decreasing contrast, this assumption was confirmed and allowed us to see the whole "flower" of a regular shape (Figure 7), with a white spot (pestle?) in the center and the surrounding petals.

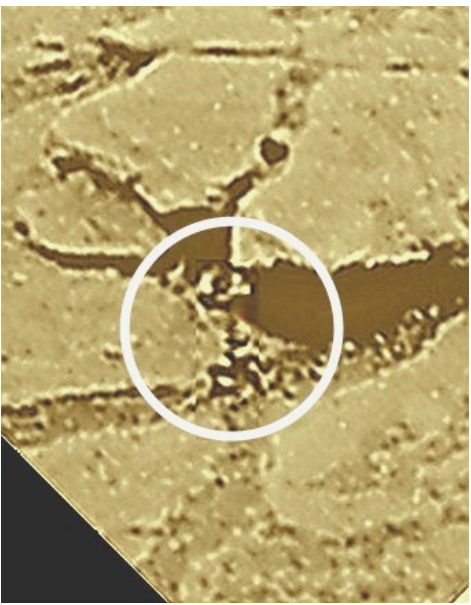

Figure 6. Processed image: "stem", "quatrefoil" at its base and the opening "burgeon", crowning the top of the "stem".

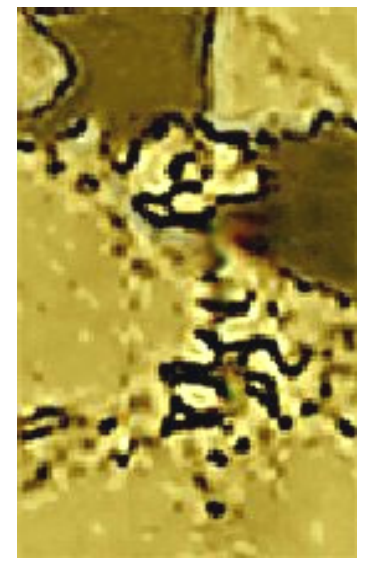

Figure 7. Flower - the same object as in Fig. 6, with lowered contrast and detailed image of the "flower", its light central part and leaves at the base. The diameter of the flower and the "quatrefoil" at the base are $5-8 \mathrm{~cm}$.

The flower is composed of six to eight light petals. Its right-hand bright part forms the triad that is repeated on all of the duplicates in Fig. 5, as part of a disclosed flower. The "flower" size is approximately the same as a "quatrefoil" at the base of the stem. The VENERA-13 panorama has been organized in such a way that Fig. 5 represents only a fragment of the black-and-white image; thus, one can talk about only the bright colors of the petals, and their color in Fig. 5 is unknown.

Another interesting but unobtrusive small bright quatrefoil was detected at the center of the VENERA-14 panoramas in a depression that is near to the landing buffer (Fig. 8, see frames 1 and 2).

In contrast to Fig. 3, its "leaves" are very bright, only slightly darker than the white cap released from the TV camera. One of the quatrefoil elements is in the shadow of a stone. The dimensions of the "leaves" are not more than $2 \mathrm{~cm}$. Despite its smaller size, the object similarity with Fig. 3 is obvious. The "stem" itself on the source panoramas (frame 1) is difficult to see; it was isolated by using a gamma-correction and in such a form is shown in column 4 as consecutive original pictures (Fig. 8, frame 3). 


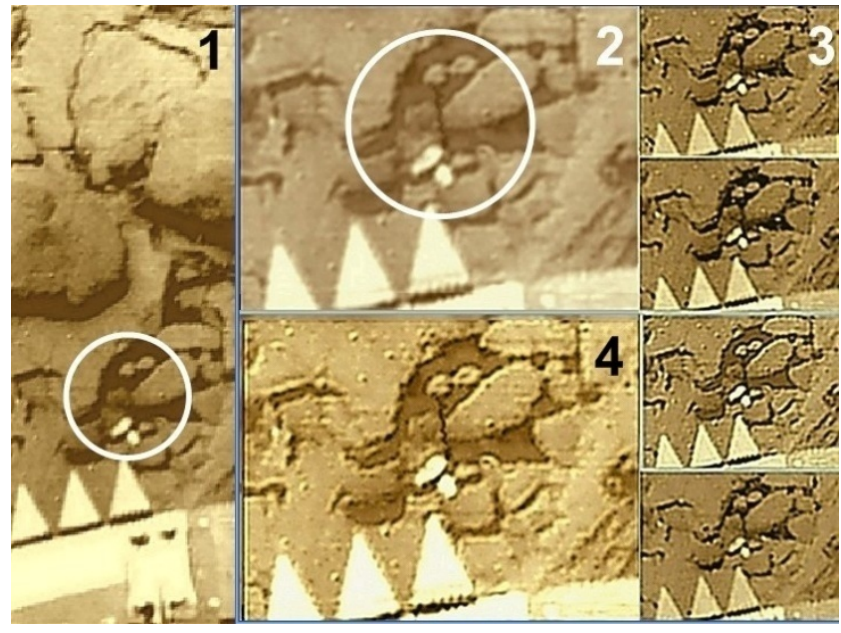

Figure 8. Stem $(1,2)$ with a bright "quatrefoil" located directly at the landing buffer of VENERA-14; its recurring images are shown on four consecutive panoramas (column 3 ). The processed image is shown in frame (4). To the right of the "flower", there is another "flower" that is visible, the stem of which is apparently situated behind the stone.

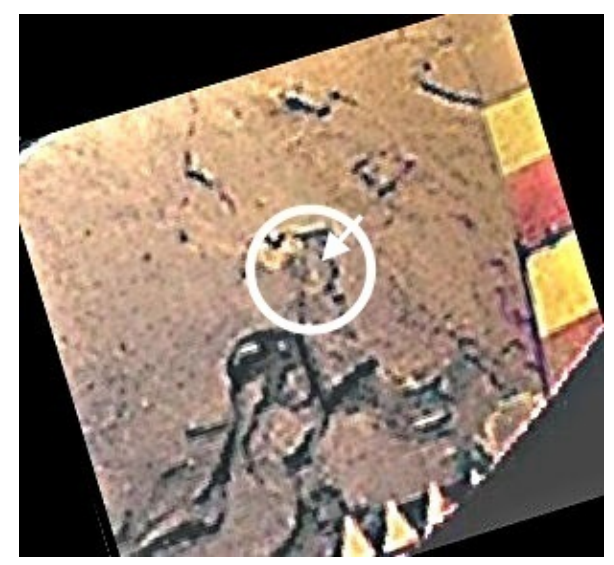

Figure 9. A possible "stem" with a light greenish colored "flower" on the VENERA-14 panorama (circled, the "flower" is marked by the arrow).

The height of the plant observed from above is approximately $10-15 \mathrm{~cm}$. There is a "flower" seen on its top, also. When the image is processed, the "stem" gets viewed as in Fig. 8, frame 4. The dimensions of the "flower" are approximately $2 \mathrm{~cm}$, also. To the right of it, another "flower" is visible, the stem of which apparently is placed behind the stone.

In Fig. 8, "stem" and "flower" are seen against the background of contrasting details and cracks in the stone slab recess. The stem rises from the recess. The object is relatively close to the camera (less than $1 \mathrm{~m}$ ), but the "flower" is small, and compared with Figure 7, the resolution is low.

Another fragment of the supposed stem (Fig. 9) refers to the panorama, for which there is a full color version. Therefore, it is possible to obtain some information about the color of the object. The initial color separation of the VENERA images cover the spectral ranges $390-510 \mathrm{~nm}$ (blue, useless due to the almost complete absorption by the atmosphere), $490-610 \mathrm{~nm}$ (green), $590-720 \mathrm{~nm}$ (red filter) and $410-800 \mathrm{~nm}$ (no filter). The solar energy distribution at the surface in the range of 410 to $800 \mathrm{~nm}$ has a maximum in the nearest infrared region. Thus, colorful panoramas can be considered conditionally as tricolor. In this sense, the flower shown in Fig. 9 that is indicated by the arrow, when compared with the background, has a greenish tint. The quatrefoil at its base in the recess is missing or not visible. However, the identification of the object in Fig. 9 is produced with the least confidence among the other figures. The flower itself, as far as can be judged by Fig. 9, is a more ephemeral feature than the objects in Fig. 7 and 8. Of course, the lack of quatrefoil at the base of the stem can be explained, for example, by attributing it to other plant species, but the spotty nature of the surface (for example, a bright elongated surface spot at the white circle top) and numerous cracks complicate the identification of the object.

\section{Discussion on the Possible Role of Burgeons and Flowers and Life on Venus}

The landing site around the landers VENERA-13 and VENERA-14 showed a significant number of vertically oriented objects that were similar to the stems of terrestrial plants. Probably, the "stems" are widespread on the planet, because the landing distances between the three different VENERA missions were 900 and $4500 \mathrm{~km}$. Both VENERA-14 cameras registered stems at opposite sides of the lander. The VENERA-9 and VENERA-13 cameras registered stems at one side only. The stems are an important complement to the objects of a hypothetical Venusian flora discussed in $[3,8,11]$. If the tops of the stems really are the burgeons and flowers, one should reflect their role. The flowers of terrestrial plants are intended for their pollination and reproduction. Pollination is conducted either by insects or by the wind. Wind-pollinated plants do not require blooms in principle, for example, the case of the poplar "fluff." Flowers attract insects. Do the tops of the stems in Fig. 7 and 8, at least indirectly, hint on the likely participants in the process of pollination?

Terramorphism of hypothetical objects of the flora and fauna of Venus was observed repeatedly in many entities [2, 8-13], for example, a terramorphic object "mushroom". Flowers with their petals in Figures 7 and 8 are new objects that are surprising to find, and it is surprising to find the occurrence of the same forms of living objects on different planets that have different physical conditions. However, what are the laws of nature that determine the recurrence of terramorphism hidden in such markedly different environments?

The high-density hypothetical habitability of Venus $[8,9]$ suggests that its surface is more similar to the bottom of a sea shoal on Earth than on the Earth's surface. If the autotrophic flora is a possible source of energy that could be used by the hypothetical fauna of the planet, then the detection of "stalks" would be corroborated. However, this autotrophic 
nature itself does not solve the problem of feeding to the fauna these rare plants. Smaller vegetation, such as grass or moss, does not appear in the VENERA pictures. Some indirect evidence of the fact that the stems and mushrooms do not exhaust the variety of small-scale flora of the planet is given in $[8,12]$. The author suggests a name "amisada" for these objects of fauna of Venus, which are found in the VENERA-14 panoramas. Consecutive images of one of the "amisadas" show that its positions are not identical. With the help of animation, it was found that amisada most likely make swinging movements, a conclusion that was arrived at by studying the surface of the stone. This motion could be a process for the purpose of searching for foods that have a much smaller dimension than the stems.

The Earth's flora began the evolution of carbon dioxide in an oxygen-free atmosphere, for which the composition was similar to the current atmosphere of Venus. As noted, the illumination on the surface of the planet Venus energetically complies with photosynthesis. Therefore, apart from the very large difference in the physical conditions, the flora of Venus should not be less rich than the Earth's flora. One can also recall the "anemas" or "mushroom" [8] in the foreground of the VENERA-13 panorama. However, the variety of forms of hypothetical fauna and flora of Venus suggests a more complex relationship among the detected objects.

The possible existence of life at conditions similar to the oxygenless $\mathrm{CO}_{2}$ atmosphere of Venus having moderately high temperatures $(735 \mathrm{~K})$ was repeatedly considered in many papers, as, for instance, life in its microbial forms. The author also considered the possibility of life evolved from the early stages of the history of the planet accommodating to conditions slowly changing to high temperatures. The $725-755 \mathrm{~K}$ temperature range near the planet's surface is, of course, absolutely incompatible with terrestrial forms of life, but thermodynamically it is no worse than the terrestrial conditions. True, the media and the existing chemical agents are unknown, but no one has looked for them. Chemical reactions at high temperatures are very active, and initial materials on Venus differ little from those on Earth $[6,7]$. There are many anaerobic mechanisms known. It seems that there are no physical prohibitions for life at high temperatures [14]. The only is "terrestrial chauvinism." Of course, photosynthesis at high temperatures and in oxygenless environment must, apparently, rely on completely different, unknown biophysical mechanisms. But what sources of energy, in principle, could be used by life in the high temperature oxygenless atmosphere, with sulfur components as main agents for meteorology? It is most natural to assume that, like on Earth, Venusian hypothetical autotrophic flora, like described above, is the source of its heterotrophic fauna. Despite the fact that the direct rays of the Sun, as a rule, do not reach the surface of the planet, there is enough light for photosynthesis. In the case of terrestrial flora scattered light, around $0.5-7 \mathrm{kLux}$, is sufficient for photosynthesis, even in the depths of dense tropical forests. The measured light on Venus is of the same order, within 3-9 kLux.

\section{Conclusion: on the Reliability of Identification of Stem Objects}

As has been noted in earlier papers, the limited angular resolution of 11' (arc minutes, VENERA-13 and VENERA-14) and 21' (VENERA-9 and VENERA-10) creates problems not only with identification but also with finding the objects being searched. Specifically, this approach concerns "stems" that are almost one-dimensional objects. A man with a very sharp eye can discern 15-20" (arc seconds) and with normal vision $30 "$. (Historically, there is a known example of the abnormal visual acuity of astronomer's William Herschel mother, who distinguished the phases of Venus with the naked eye). Thus, the resolution of the panoramas of Venus exceeds normal vision by 22 times. The methods of treatment of a single low-noise image can increase its clarity by a few times. Because the panoramas duplicate images up to eight times, it is possible not only to eliminate some of the random noise but also to increase further the resolution by slightly offset images when they are received. Unfortunately, along with the displacement, the image is distorted due to the rapid heating of the equipment of the VENERA landers. Therefore, we first must identify a "suspicious" object and then perform batch processing of the images.

It should be mentioned specifically how it is possible to notice a suspicious object itself. This process plays a major role in the vigilance and attention of a researcher. For example, in the input panorama (Fig. 2), a "stem" shown in Fig. 3 resembles only a thin scratch, but it is repeated at all panoramas and in the same place. Care is also needed to distinguish an object from its background in shades of gray or color (for color images) and to attempt to understand what a suspicious object looks like. The ability to notice unusual objects is basically related to carefulness but requires other skills. (A well-known example of attention is the ability to notice a distant ship on the sea's horizon. Some people can notice it and others do not.) The authors suggested that the ability to notice an unusual shape could be enhanced at artists. However, this suggestion was an error.

The clarity of a picture element that has a fixed size depends on the distance. The line resolution was 211 pixels and 11' (arc min); thus, a pixel size of $0.5 \mathrm{~cm}$ (thickness of the stem) will correspond to the distance $0.005 /(11 / 3438)=$

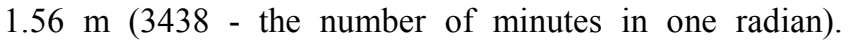
Unfortunately, upon heating, the equipment's adjustment deteriorated, and the actual resolution became worse. If the image of a specific object is not single, as in the case of stems, then batch processing and stacking can be used to study the details.

In Fig. 3, the knots on the stem have a 2-3 pixel size (1-2 $\mathrm{cm}$ ), and the "bud" has a 5-6 cm size. Based on the geometry of the resulting corners, we can assume that each point of the image of a stem that is at a distance of $3 \mathrm{~m}$ is eroded by four pixels, and its contrast is reduced by about half (due to the one-dimensional structure of the object). A definite advantage arises from batch processing of images (stacking 
including). For more remote stems, the contrast is reduced; thus, their detection becomes impossible.

\section{REFERENCES}

[1] L.V. Ksanfomality. Possible Detection of Life on the Planet Venus. Doklady Physics, Vol. 57, No. 9, pp. 367-372, 2012.

[2] L.V. Ksanfomality. Mobility and Other Features of Hypothetical Venusian Fauna. Doklady Physics, Vol. 57, No.12, pp. 497-501. 2012.

[3] L.V. Ksanfomality. Possible Signs of Life on the Planet Venus. International Journal of Astronomy and Astrophysics (IJAA), Vol. 3, pp. 57-79, 2013.

[4] A.S. Selivanov, Yu.M. Gektin, M.A. Gerasimov. Continuation of the TV Investigation of Venus surface by means of landers. Kosmich. Issled., Vol. 21, pp.176-182, 1983.

[5] B. E. Moshkin, A. P. Economov, V. I. Moroz et al., Spectrophotometric Experiment on VENERA-13, -14 Landers. Kosmich. Issled., Vol. 21, No. 2, pp. 236-245, 1983.

[6] L.V. Ksanfomality. The Planet Venus. FIZMATLIT, Moscow, 1985, 376 p. (in Russian).
[7] Venus. D. M. Hunten, L. Colin, T. M. Donahue and V.I. Moroz, Eds., , University of Arizona Press, Tucson, 1983.

[8] L.V. Ksanfomality. Snake - a new object of hypothetical fauna of Venus. Doklady Physics, Vol. 58, No 4, pp. 395-400, 2013.

[9] L.V. Ksanfomality. Hypothetical life detected on the planet Venus. International Letters of Chemistry, Physics and Astronomy (ILSPA), Vol.10, pp.76-89, 2013.

[10] L.V. Ksanfomality. Looking for Hypothetical Flora on Venus // Advances in Zoology and Botany, Vol.1, No 1, pp. 7-16, 2013.

[11] L.V. Ksanfomality. An object of assumed Venusian flora. Doklady Physics. Vol.58, No 5, pp. 204-206, 2013.

[12] L.V. Ksanfomality. Quest for hypothetic fauna of Venus at the VENERA-14 landing site: Amisadas. Doklady Physics, Vol.58, No 3, pp. 272-276, 2013.

[13] L.V. Ksanfomality. Looking for Mobility of Hypothetical Venusian Fauna Advances in Zoology and Botany, Vol.1, No 2, pp. 25-29, 2013.

[14] A. S. Spirin. Comment to the Article L. V. Ksanfomality Venus as a Natural Laboratory for Search of Life in High Temperature Conditions: Events on the Planet on March 1, 1982. Solar System Research, Vol. 46, No. 5, 2012, p. 386. 